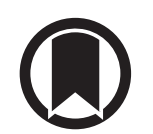

CrossMark

\title{
The European Multicentre Bronchiectasis Audit and Research Collaboration (EMBARC) ERS Clinical Research Collaboration
}

\author{
Stefano Aliberti (101, Eva Polverino², James D. Chalmers ${ }^{3}$, Josje Altenburg ${ }^{4}$, \\ Michal Shteinberg ${ }^{5}$, Pieter C. Goeminne 6,7 , Tobias Welte ${ }^{8}$, Amelia Shoemark ${ }^{3}$, \\ Marta Almagro 9 and Francesco Blasi ${ }^{1}$ on behalf of the EMBARC Clinical \\ Research Collaboration
}

\begin{abstract}
Affiliations: ${ }^{1}$ Fondazione IRCCS Cà Granda Ospedale Maggiore Policlinico, Respiratory Unit and Adult Cystic Fibrosis Center, Dept of Pathophysiology and Transplantation, University of Milan, Milan, Italy. ${ }^{2}$ Respiratory Disease Dept, Hospital Universitari Vall d'Hebron (HUVH) Institut de Recerca Vall d'Hebron (VHIR) Passeig Vall d'Hebron, CIBERES, Barcelona, Spain. ${ }^{3}$ Scottish Centre for Respiratory Research, University of Dundee, Dundee, UK. ${ }^{4}$ Dept of Pulmonary Diseases, Amsterdam University Medical Center, Amsterdam, The Netherlands. ${ }^{5}$ Pulmonary Institute and CF Center, Carmel Medical Center, Haifa, Israel. ${ }^{6}$ Respiratory Medicine, University Hospital Gasthuisberg, Leuven, Belgium. ${ }^{7}$ Respiratory Disease, AZ Nikolaas, SintNiklaas, Belgium. ${ }^{8}$ Dept of Respiratory Medicine, Hannover Medical School, Hannover, Germany. ${ }^{9}$ EMBARC/ ELF bronchiectasis patient advisory group, European Lung Foundation, Sheffield, UK.
\end{abstract}

Correspondence: Stefano Aliberti, Fondazione IRCCS Cà Granda Ospedale Maggiore Policlinico, Respiratory Unit and Adult Cystic Fibrosis Center, Dept of Pathophysiology and Transplantation, University of Milan, Via Francesco Sforza 35, Milan 20122, Italy. E-mail: stefano.alibertiaunimi.it

@ERSpublications

The European Multicentre Bronchiectasis Audit and Research Collaboration (EMBARC) is the ERS Clinical Research Collaboration born to develop scientific, educational and advocacy activities for bronchiectasis at an international level http://ow.ly/EHr030mxEcC

Cite this article as: Aliberti S, Polverino E, Chalmers JD, et al. The European Multicentre Bronchiectasis Audit and Research Collaboration (EMBARC) ERS Clinical Research Collaboration. Eur Respir J 2018; 52 : 1802074 [https://doi.org/10.1183/13993003.02074-2018].

The European Multicentre Bronchiectasis Audit and Research Collaboration (EMBARC) was established in 2012 as a collaborative group within the Respiratory Infections Assembly of the European Respiratory Society (ERS) with the objective of creating a European bronchiectasis registry, harmonising existing databases, and identifying opportunities to raise the profile of bronchiectasis at an international level [1].

Bronchiectasis has been always described as one of the most neglected respiratory diseases, with no licensed treatments in the face of a substantial burden in terms of poor patient quality of life and increasing healthcare costs $[2,3]$. Although the label of "orphan disease" does not apply to bronchiectasis in light of recent epidemiological data, it has been used historically to reflect the lack of interest that scientists, clinicians, regulators and funders had in this disease until one decade ago. As an example, the recommendations from the British guidelines on bronchiectasis published in 2010 were almost exclusively based on expert opinion because of the absence of high-quality evidence [4].

The EMBARC project was born within an environment where many doubted whether bronchiectasis was really a disease, and where most management was based on paradigms developed in cystic fibrosis or 
chronic obstructive pulmonary disease. Research into bronchiectasis was limited and came mainly from the UK, Spain, the USA and Australia. A lack of European patient organisations meant the absence of a clear voice from patients suffering from this disease. Funders did not recognise bronchiectasis as a problem and so rarely provided funding for bronchiectasis research and, finally, there were no internationally recognised standards of care.

6 years since its establishment, EMBARC is now recognised as one of the most successful Clinical Research Collaborations (CRCs) within the ERS [5]. EMBARC has been supported by a large and enthusiastic community of more than 150 centres in more than 40 countries. One of the keys to its success has been the support of the European Lung Foundation, which established a European advisory group of people with bronchiectasis and their caregivers, providing the first "patient voice" for bronchiectasis in Europe, and all EMBARC projects and meetings now involve patient representatives [6]. With the support of international partners and the ERS, the profile of bronchiectasis in Europe and the available evidence have improved. In this article, we list some of the achievements of the EMBARC project.

EMBARC was approved as an ERS CRC in 2013 with the primary objective of developing a pan-European, prospective registry of adults with bronchiectasis following the recommendations from the European Union Committee of Experts on Rare Diseases (study website: www.bronchiectasis.eu). The detailed protocol was published in 2016 [7]. More than 14000 patients had been recruited up to September 2018, with up to 4-year follow-up now available for some patients. Research priorities in bronchiectasis were identified in the early phase of the CRC by a Europe-wide group of experts and patients/carers (the EMBARC Roadmap Study Group), with the aim of guiding collaborative research and supporting international stakeholders in designing calls for funding in the field [8]. EMBARC also provided consensus guidance on cross-infection issues in bronchiectasis and led a consensus working group of international experts who worked together for $>1$ year to develop a simple and operational definition of bronchiectasis exacerbation for use in clinical trials $[9,10]$. Further consensus documents on the definitions of bronchiectasis, chronic infection and eradication are planned for 2018 and beyond, with the intention that these will be used in planning clinical trials. More than 10 datasets from different European countries have been aligned and designated as the FRIENDS dataset (Facilitating Research Into Existing National DataSets). Their analysis ended up with $>10$ original publications evaluating disease severity, ageing, radiology, aetiologies, phenotypes and endotypes, the role of Pseudomonas and exacerbations, and the impact of comorbidities in bronchiectasis [11-23]. EMBARC supported the development of an international task force of experts and patients who published in September 2017 the ERS guidelines for the management of adult bronchiectasis [24]. In line with the fact that the registry can provide crucial insights in planning and executing clinical trials, not only in terms of feasibility but also in providing patient input into the trial design and validation of end-points, EMBARC is currently supporting three randomised controlled trials on new drugs in bronchiectasis (one is registered at ClinicalTrials.gov with identifier NCT03696290) and further trials, including pragmatic ones, are planned. EMBARC promoted three international conferences specifically focused on bronchiectasis, which have been held in Hannover (Germany) in 2016, Milan (Italy) in 2017 and Washington, DC (USA) in 2018. In 2019, EMBARC will host an ERS research seminar in Barcelona (Spain) jointly with the ERS CRC SHARP (Severe Heterogeneous Asthma Research collaboration, Patient-centred), addressing the overlap between bronchiectasis and airways disease [25]. Finally, other investigators from several other countries outside of Europe are developing bronchiectasis research infrastructures using the EMBARC platform; India and Australia are already working collaboratively with EMBARC (with their respective websites at https:// hicservices.dundee.ac.uk/rrni and https://lungfoundation.com.au/research/our-research/bronchiectasis/). EMBARC is also central to the European Reference Network (ERN) for rare respiratory diseases (ERN-LUNG) core network for bronchiectasis [26]. Some of the most successful initiatives developed by EMBARC are represented in figure 1.

Although important achievements, especially from a clinical and epidemiological point of view, were reached during the first 3 years of EMBARC, some important research priorities still remain unexplored. Heterogeneity of the disease remains the greatest clinical challenge. There are no licensed therapies for bronchiectasis and clinical trials in bronchiectasis have been inconsistent, perhaps because we have not yet identified the endotypes of bronchiectasis that respond to specific therapies such as inhaled antibiotics, macrolides and mucoactive drugs [27, 28]. Translational and cooperative studies will be needed in the future to investigate the pathophysiology of bronchiectasis, and to identify treatment response endotypes. Some aspects of bronchiectasis, including inflammatory pathways, the microbiome, proteomics and genomics, are not sufficiently studied. Their investigation on a large scale could help to better understand bronchiectasis pathophysiology, to better phenotype patients and individualise their management.

A second phase of EMBARC as an ERS CRC (namely EMBARC2) has been funded from 2017 to 2020 and is keen to have on board the highest number of ERS members possible. EMBARC2 aims to phenotype 


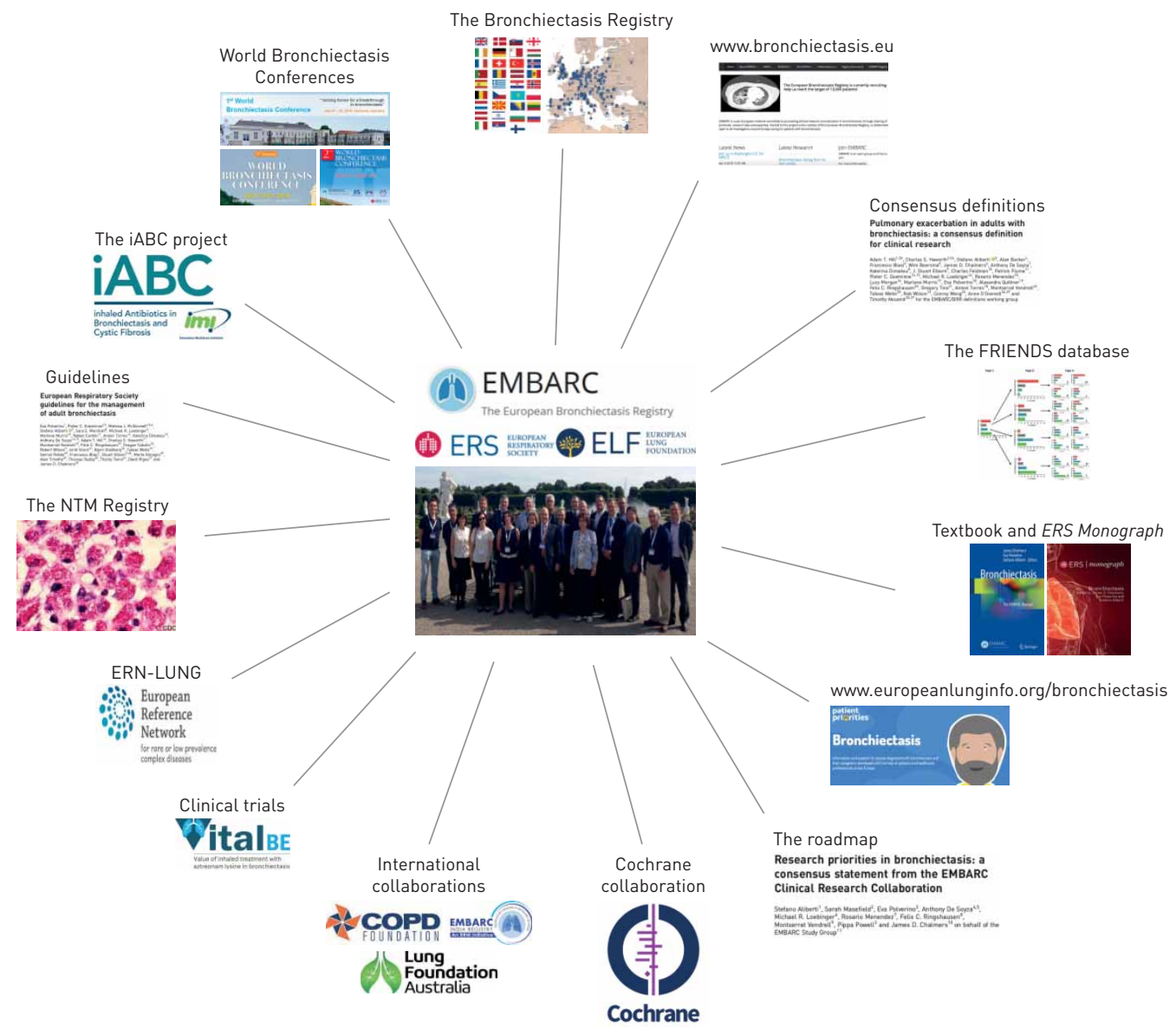

FIGURE 1 Initiatives developed by the European Multicentre Bronchiectasis Audit and Research Collaboration (EMBARC) up to 2018. iABC: Inhaled Antibiotics in Bronchiectasis and Cystic Fibrosis; NTM: nontuberculous mycobacteria; ERN: European Reference Network; FRIENDS: Facilitating Research Into Existing National DataSets.

and endotype bronchiectasis, developing strategies for more successful clinical trials and personalised medicine, and for reducing disease heterogeneity. The core of this project will be the development of an international bioresource, using the registry as a backbone to build a repository of blood, DNA, sputum and other biological materials for use in translational research. This project will include a deep evaluation of local and systemic inflammation, microbiota and genomics in bronchiectasis, in order to address relevant research questions on its pathophysiology. EMBARC2 has also the ambition to generate recommendations for design and conduct of bronchiectasis trials, for end-points to be used in those trials and for the research priorities in each area.

Specific work packages have been designed on the research priorities identified previously, including the European Bronchiectasis Registry, the EMBARC-BRIDGE study, the Bronchiectasis and Airways Disease "overlap" and on clinical trials support and feasibility. The role of nontuberculous mycobacteria in the pathogenesis and progression of bronchiectasis will also be a key topic [29, 30]. The BRIDGE (Bronchiectasis Research Involving Databases, Genomics and Endotyping to match the right treatment to the right patient) study will be an observational, cohort study aiming at determining the molecular endotypes of bronchiectasis during the stable state, as well as the causes and inflammatory profiles of exacerbations, validating biomarkers to use in stratified medicine, and performing in vivo or in vitro proof-of-concept studies using phenotypic data to identify patient populations likely to benefit in future randomised controlled trials. The kick-off meeting of work package leaders and EMBARC2 management board was recently held in Paris (France) during the ERS International Congress.

EMBARC has made an important contribution to bronchiectasis research, care and increasing the awareness of a disease that is no longer an "orphan". Over the next 3 years, EMBARC2 will make a substantial contribution to the epidemiology, clinical and translational research, advocacy and education in bronchiectasis and we open a call to all the ERS members to be part of that. 
Conflict of interest: S. Aliberti reports grants and personal fees from Bayer Healthcare, Aradigm Corporation, Grifols, Chiesi and Insmed, and personal fees from Astra Zeneca, Basilea, Zambon, Novartis, Raptor, Actavis UK Ltd and Horizon, all outside the submitted work. E. Polverino reports personal consultancy and speaker fees from Bayer, Grifols and Chiesi, consultancy fees from Insmed, speaker fees from Teva and Zambon, and research grants from Chiesi, all outside the submitted work. J.D. Chalmers reports grants from Bayer Healthcare, Insmed, Zambon, Novartis, Chiesi and Grifols, during the conduct of the study. He also reports research grants for COPD from GlaxoSmithKline, Boehringer Ingelheim, AstraZeneca and Pfizer; grants for research into bronchiectasis from Bayer Healthcare and Grifols; grants from Insmed; and personal fees from GlaxoSmithKline, Boehringer-Ingelheim, Pfizer, Bayer Healthcare, Grifols, Napp (consulting), Aradigm corporation (consulting) and Insmed, all outside the submitted work. J. Altenburg has nothing to disclose. M. Shteinberg reports research grants from GSK, Novartis, Trudell pharma and GSK; travel grants from Novartis, Actelion, Boehringer Ingelheim, GSK and Rafa; speaker's fees from Boehringer Ingelheim, GSK, AstraZeneca, Teva and Novartis; and advisory fees from GSK, Boehringer Ingelheim, Kamada and Horizon pharma. P.C. Goeminne reports personal fees for lectures from AstraZeneca and Chiesi, and non-financial support to attend conferences from Chiesi and Boehringer, outside the submitted work. T. Welte reports grants from Novartis, Pfizer, Bayer, Grifols and Insmed, and personal fees for lectures and advisory boards from AstraZeneca, Bayer, Boehringer, GSK, MSD, Novartis and Pfizer, al outside the submitted work. A. Shoemark has nothing to disclose. M. Almagro reports non-financial support from Polyphor, outside the submitted work. F. Blasi reports grants and personal fees from AstraZeneca, Bayer and Pfizer, and personal fees from Chiesi, GSK, Guidotti, Insmed, Menarini, Novartis and Zambon, all outside the submitted work.

Support statement: EMBARC2 is supported by the European Respiratory Society through the Clinical Research Collaboration. The project is supported by project partners: Chiesi, Grifols, Insmed, Novartis and Zambon. EMBARC is supported by European Union/European Federation of Pharmaceutical Industries and Associations (EFPIA) Innovative Medicines Initiative (IMI) Inhaled Antibiotics in Bronchiectasis and Cystic Fibrosis (iABC) grant agreement number 115721.

\section{References}

1 Chalmers JD, Crichton M, Goeminne PC, et al. The European Multicentre Bronchiectasis Audit and Research Collaboration (EMBARC): experiences from a successful ERS Clinical Research Collaboration. Breathe 2017; 13: $180-192$.

2 Chalmers JD, Aliberti S, Blasi F. Management of bronchiectasis in adults. Eur Respir J 2015; 45: 1446-1462.

3 Chalmers JD, Loebinger M, Aliberti S. Challenges in the development of new therapies for bronchiectasis. Expert Opin Pharmacother 2015; 16: 833-850.

4 Pasteur MC, Bilton D, Hill AT. British Thoracic Society guideline for non-CF bronchiectasis. Thorax 2010; 65: Suppl. 1, i1-i58.

5 Brightling C, Genton C, Bill W, et al. ERS Clinical Research Collaborations: underpinning research excellence. Eur Respir J 2018; 52: 1801534.

6 Chalmers JD, Timothy A, Polverino E, et al. Patient participation in ERS guidelines and research projects: the EMBARC experience. Breathe 2017; 13: 194-207.

7 Chalmers JD, Aliberti S, Polverino E, et al. The EMBARC European Bronchiectasis Registry: protocol for an international observational study. ERJ Open Res 2016; 2: 00081-2015.

8 Aliberti S, Masefield S, Polverino E, et al. Research priorities in bronchiectasis: a consensus statement from the EMBARC Clinical Research Collaboration. Eur Respir J 2016; 48: 632-647.

9 Chalmers JD, Ringshausen FC, Harris B, et al. Cross-infection risk in patients with bronchiectasis: a position statement from the European Bronchiectasis Network (EMBARC), EMBARC/ELF patient advisory group and European Reference Network (ERN-Lung) Bronchiectasis Network. Eur Respir J 2018; 51: 1701937.

10 Hill AT, Haworth CS, Aliberti S, et al. Pulmonary exacerbation in adults with bronchiectasis: a consensus definition for clinical research. Eur Respir J 2017; 49: 1700051.

11 Chalmers JD, Goeminne P, Aliberti S, et al. The bronchiectasis severity index. An international derivation and validation study. Am J Respir Crit Care Med 2014; 189: 576-585.

12 McDonnell MJ, Aliberti S, Goeminne PC, et al. Multidimensional severity assessment in bronchiectasis: an analysis of seven European cohorts. Thorax 2016; 71: 1110-1118.

13 Finch S, McDonnell MJ, Abo-Leyah $\mathrm{H}$, et al. A comprehensive analysis of the impact of Pseudomonas aeruginosa colonization on prognosis in adult bronchiectasis. Ann Am Thorac Soc 2015; 12: 1602-1611.

14 Lonni S, Chalmers JD, Goeminne PC, et al. Etiology of non-cystic fibrosis bronchiectasis in adults and its correlation to disease severity. Ann Am Thorac Soc 2015; 12: 1764-1770.

15 Araújo D, Shteinberg M, Aliberti S, et al. Standardised classification of the aetiology of bronchiectasis using an objective algorithm. Eur Respir J 2017; 50: 1701289.

16 Aliberti S, Lonni S, Dore S, et al. Clinical phenotypes in adult patients with bronchiectasis. Eur Respir J 2016; 47: $1113-1122$.

17 Chalmers JD, McDonnell MJ, Rutherford R, et al. The generalizability of bronchiectasis randomized controlled trials: a multicentre cohort study. Respir Med 2016; 112: 51-58.

18 Bellelli G, Chalmers JD, Sotgiu G, et al. Characterization of bronchiectasis in the elderly. Respir Med 2016; 119: 13-19.

19 McDonnell MJ, Aliberti S, Goeminne PC, et al. Comorbidities and the risk of mortality in patients with bronchiectasis: an international multicentre cohort study. Lancet Respir Med 2016; 4: 969-979.

20 De Soyza A, McDonnell MJ, Goeminne PC, et al. Bronchiectasis rheumatoid overlap syndrome is an independent risk factor for mortality in patients with bronchiectasis: a multicenter cohort study. Chest 2017; 151: 1247-1254.

21 Bedi P, Chalmers JD, Goeminne PC, et al. The BRICS (Bronchiectasis Radiologically Indexed CT Score): a multicenter study score for use in idiopathic and postinfective bronchiectasis. Chest 2018; 153: 1177-1186.

22 Chalmers JD, Aliberti S, Filonenko A, et al. Characterization of the "frequent exacerbator phenotype" in bronchiectasis. Am J Respir Crit Care Med 2018; 197: 1410-1420.

23 Araújo D, Shteinberg M, Aliberti S, et al. The independent contribution of Pseudomonas aeruginosa infection to long-term clinical outcomes in bronchiectasis. Eur Respir J 2018; 51: 1701953. 
24 Polverino E, Goeminne PC, McDonnell MJ, et al. European Respiratory Society guidelines for the management of adult bronchiectasis. Eur Respir J 2017; 50: 1700629.

25 Polverino E, Dimakou K, Hurst J, et al. The overlap between bronchiectasis and chronic airway diseases: state of the art and future directions. Eur Respir J 2018; 52: 1800328.

26 Humbert M, Wagner TO. Rare respiratory diseases are ready for primetime: from Rare Disease Day to the European Reference Networks. Eur Respir J 2017; 49: 1700085.

27 De Soyza A, Aksamit T, Bandel TJ, et al. RESPIRE 1: a phase III placebo-controlled randomised trial of ciprofloxacin dry powder for inhalation in non-cystic fibrosis bronchiectasis. Eur Respir J 2018; 51: 1702052.

28 Aksamit T, De Soyza A, Bandel TJ, et al. RESPIRE 2: a phase III placebo-controlled randomised trial of ciprofloxacin dry powder for inhalation in non-cystic fibrosis bronchiectasis. Eur Respir J 2018; 51: 1702053.

29 Faverio P, Stainer A, Bonaiti G, et al. Characterizing non-tuberculous mycobacteria infection in bronchiectasis. Int J Mol Sci 2016; 17: E1913.

30 Aliberti S, Codecasa LR, Gori A, et al. The Italian registry of pulmonary non-tuberculous mycobacteria - IRENE: the study protocol. Multidiscip Respir Med 2018; 13: Suppl. 1, 33. 